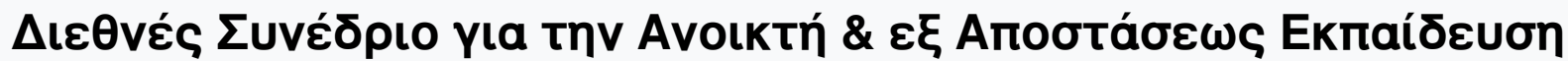

Tóp. 6, Ap. 2B (2011)

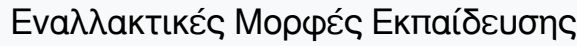

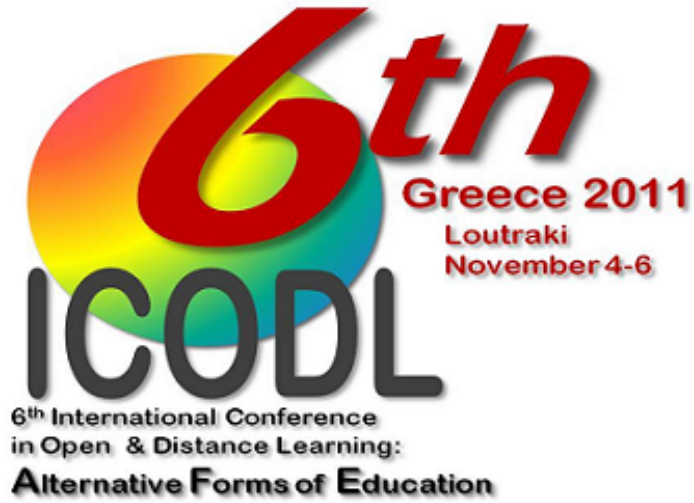

TOMOE B

PART / MEPOE B

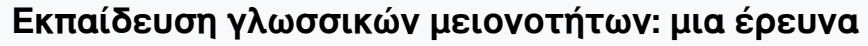

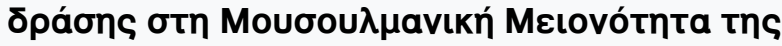
Өpákns

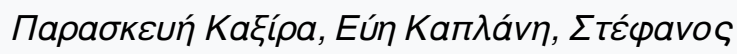
Makpuyıávvns, Гıávvns Пoutaxíons

doi: $10.12681 /$ icodl. 664 


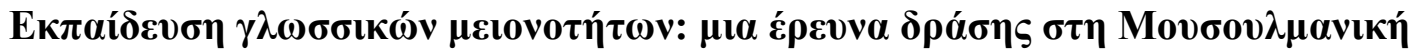

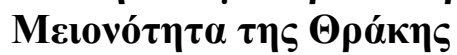

\section{Educating of linguistic minorities: an action research in the Muslim Minority of Thrace}

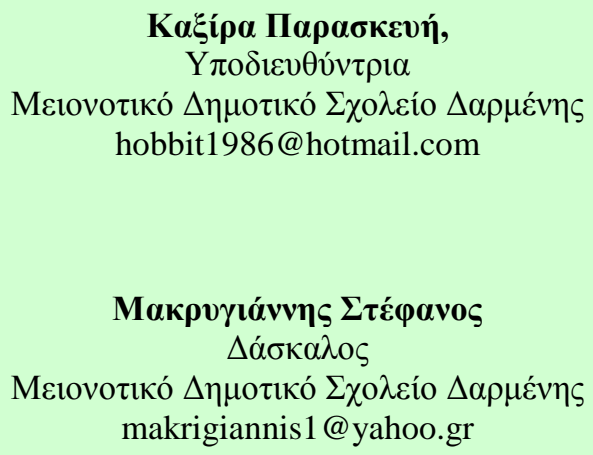

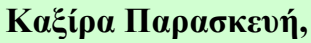

Y $\pi 0 \delta 1 \varepsilon v \theta v ́ v \tau \rho 1 \alpha$

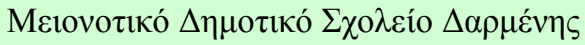
hobbit1986@hotmail.com

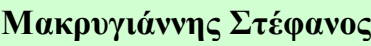
$\Delta \alpha ́ \sigma \kappa \alpha \lambda \circ \varsigma$

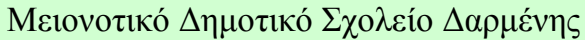
makrigiannis1@yahoo.gr

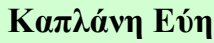 \\ MA

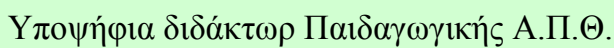 \\ $\Delta \alpha \sigma \kappa \alpha ́ \lambda \alpha$

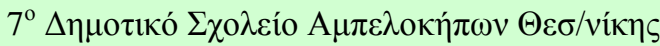 \\ eve_kaplani@pathfinder.gr

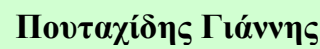 \\ MA \\ $\Theta \varepsilon \alpha \tau \rho о \pi \alpha \imath \delta \alpha \gamma \omega \gamma o ́ s$

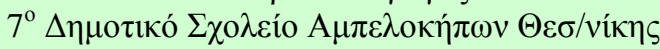 \\ mgrjanis@hotmail.com
}

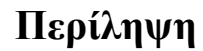

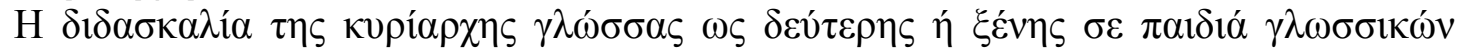

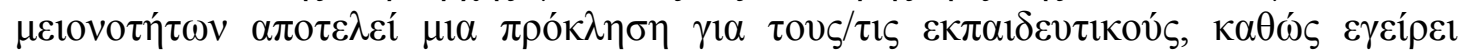

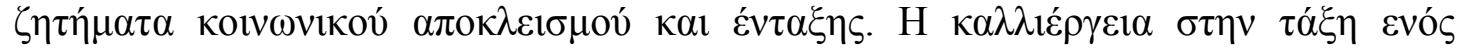

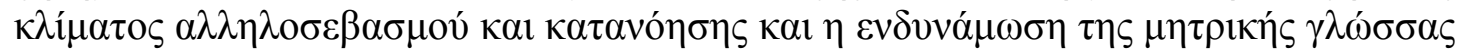

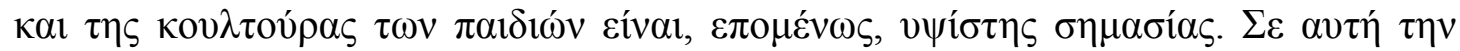

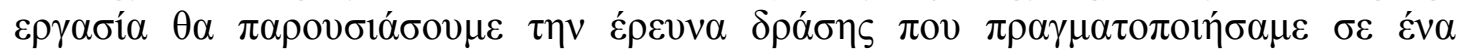

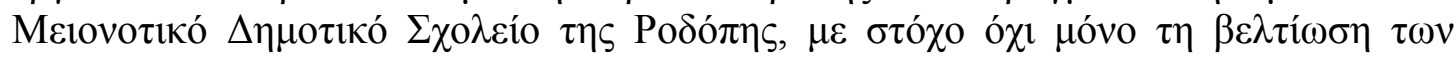

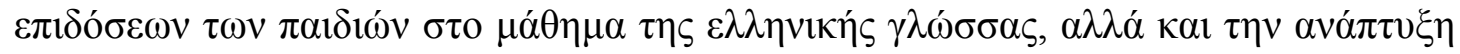

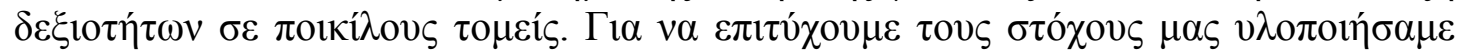

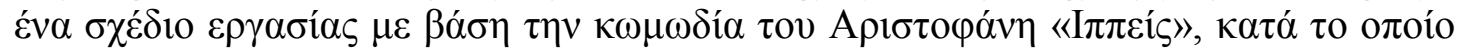

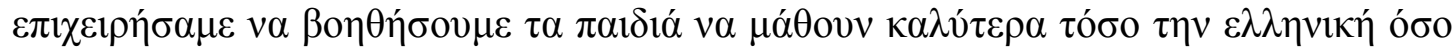

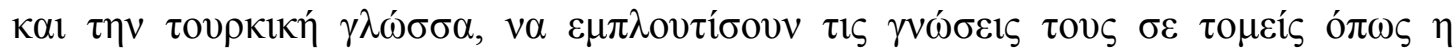

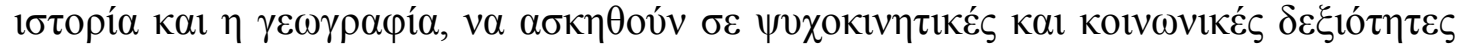

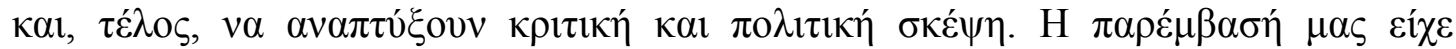

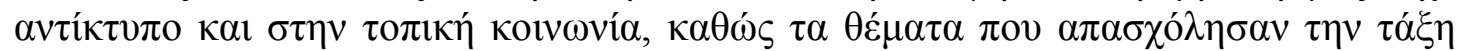

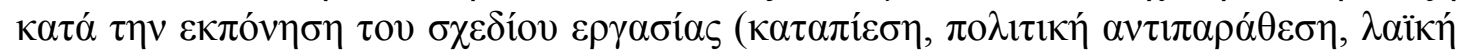

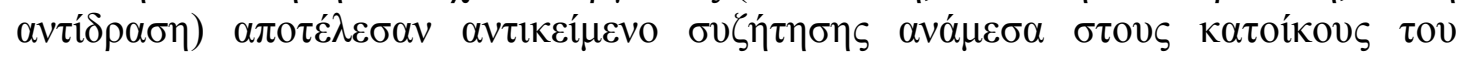

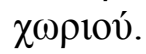

\begin{abstract}
In this paper we present an action research that took place in a Muslim Minority Primary School of Rhodopi. The project that was held in order to enhance the students' school achievement and critical thought was based on Aristophanes' "Ippeis". The research also had an impact on the village's society.
\end{abstract}

Key-words: minority education, action research, forum theatre 


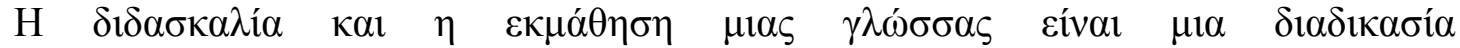

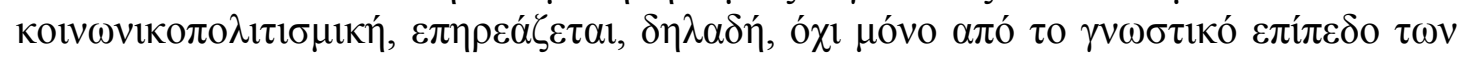

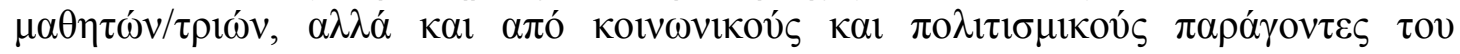

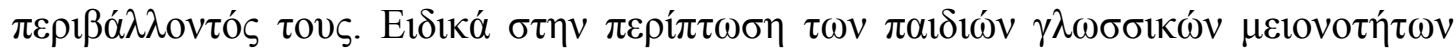

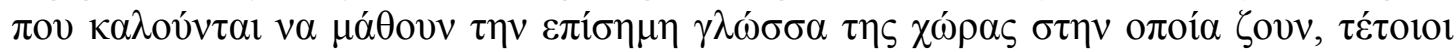

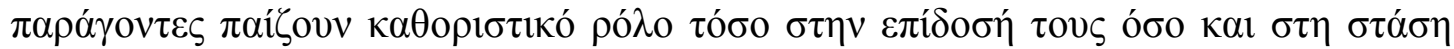

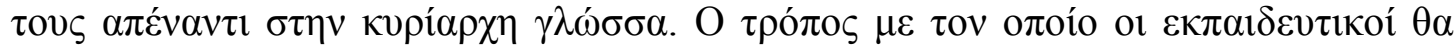

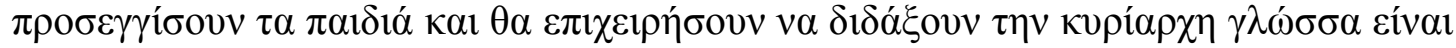

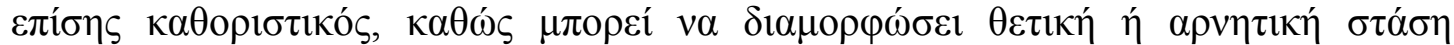

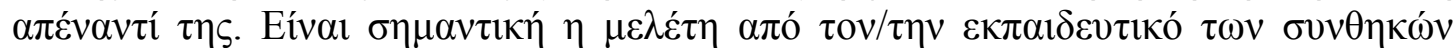

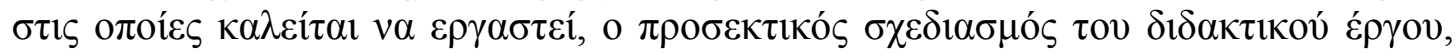

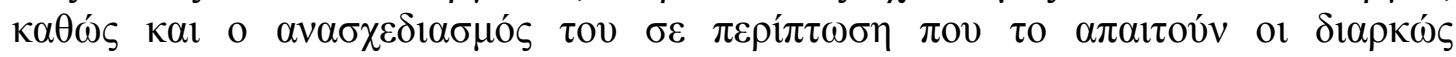
$\alpha v \alpha \delta 1 \alpha \mu о \rho \varphi \omega v o ́ \mu \varepsilon v \varepsilon \varsigma$ $\sigma v v \theta \eta \dot{\kappa} \kappa \varepsilon$ (Altrichter et al., 2001).

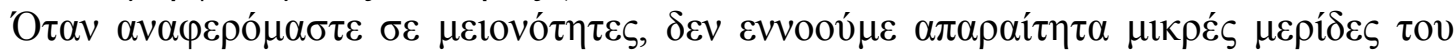

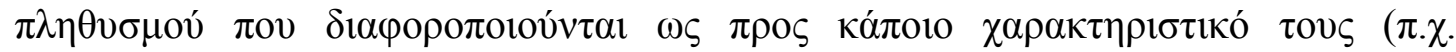

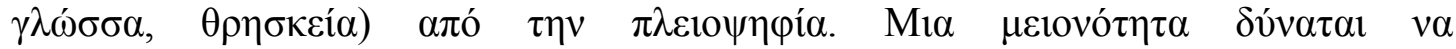

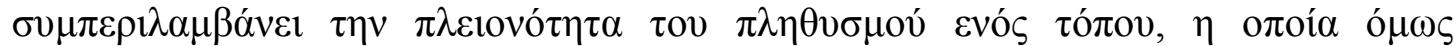

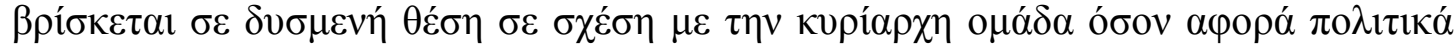

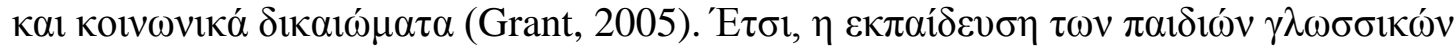

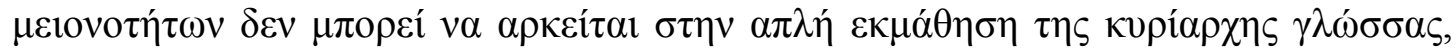

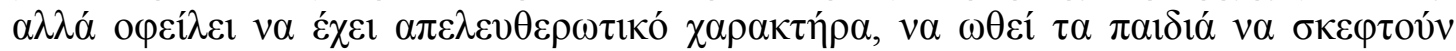

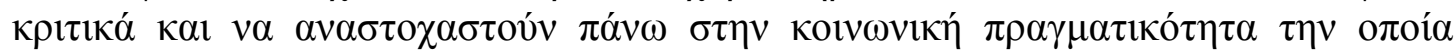
ßıஸ́vovv (Freire, 1977, 1996)

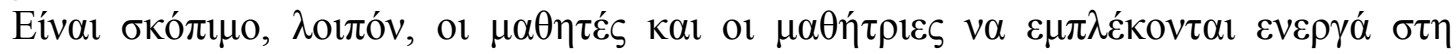

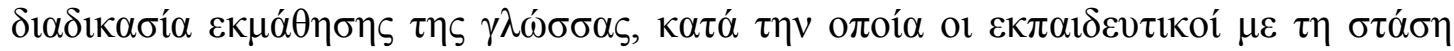

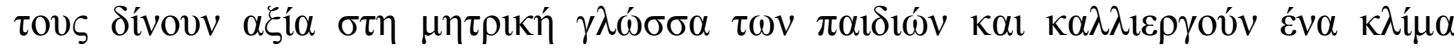

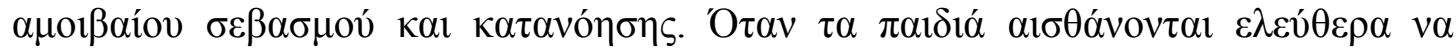

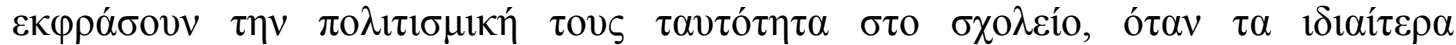

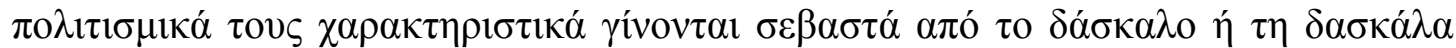

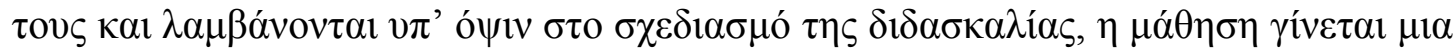

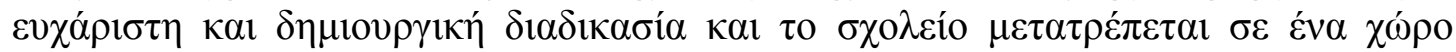

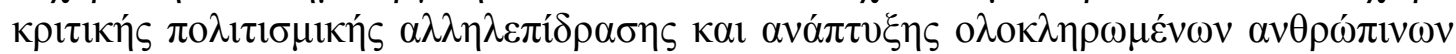

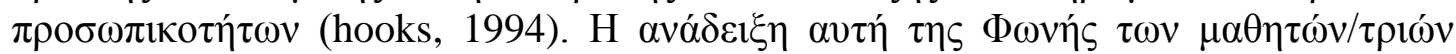

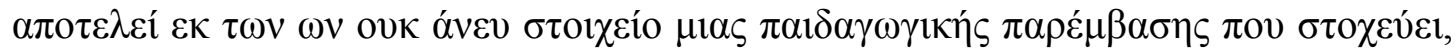

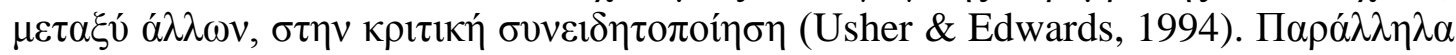

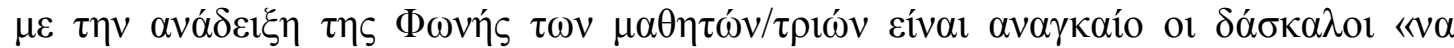

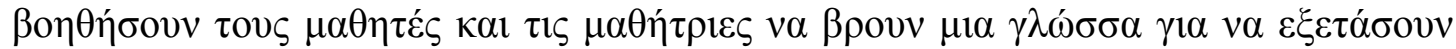

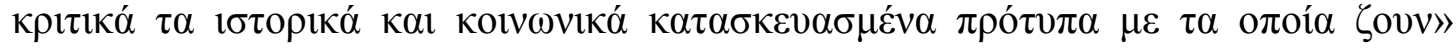

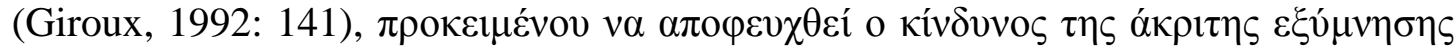

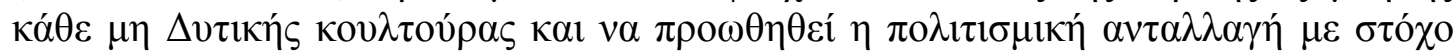
$\tau \eta v \kappa o เ v \omega v i \kappa \eta ́ ~ \alpha v \alpha ́ \pi \tau v \xi \eta$.

\section{H épevva}

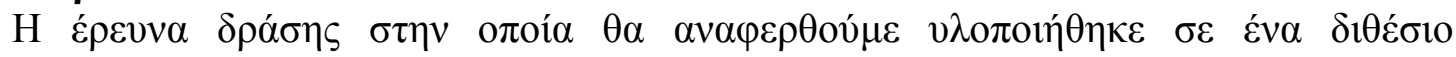

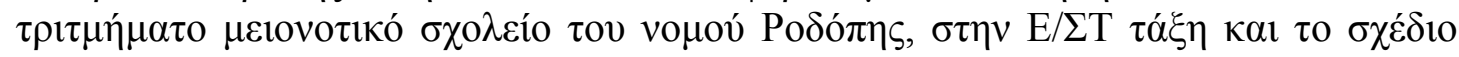

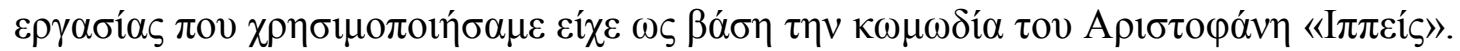

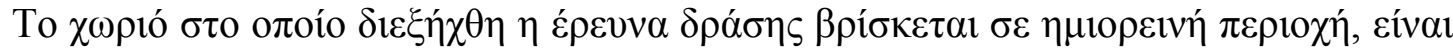

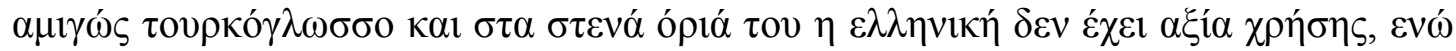

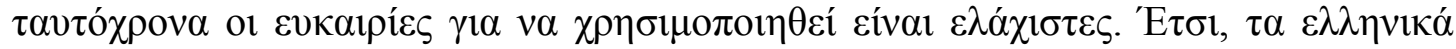




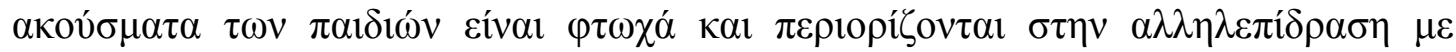

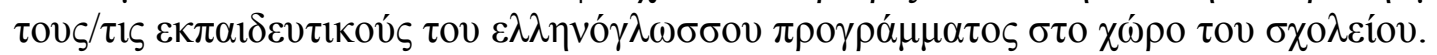

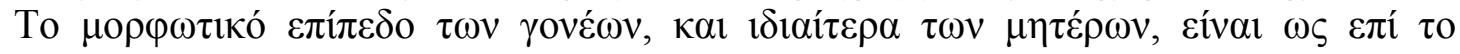

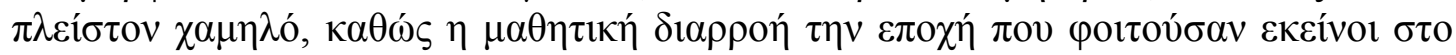

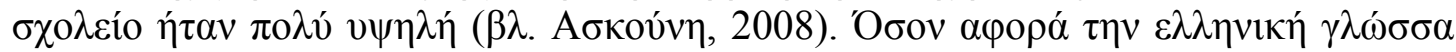

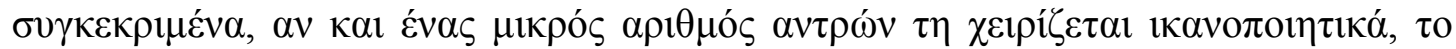

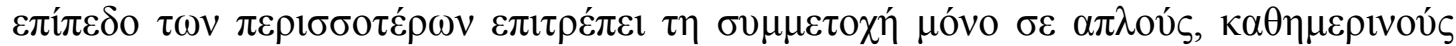

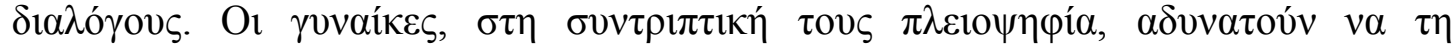

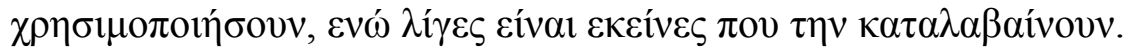

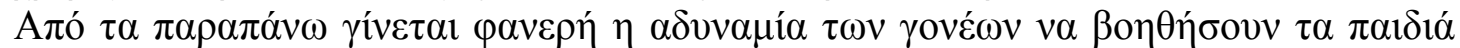

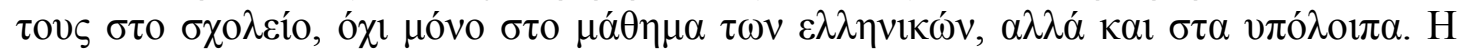

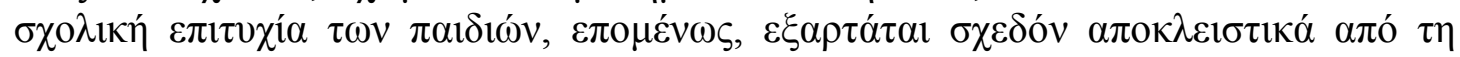

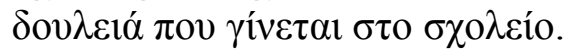

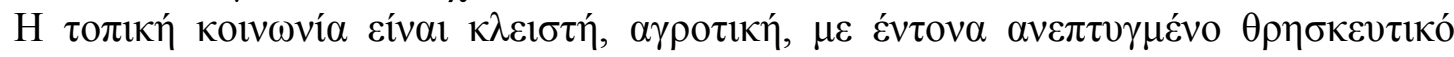

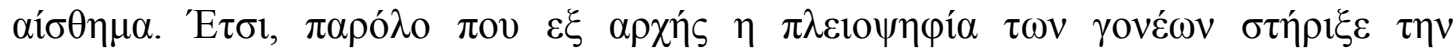

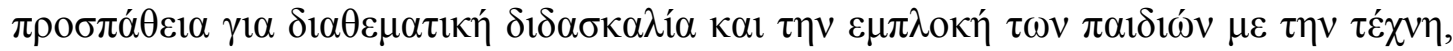

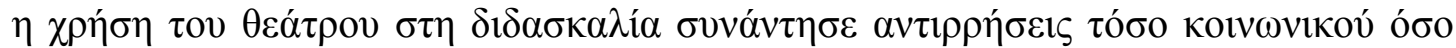

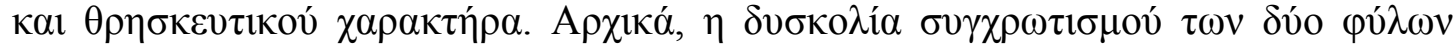

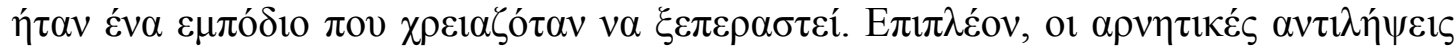

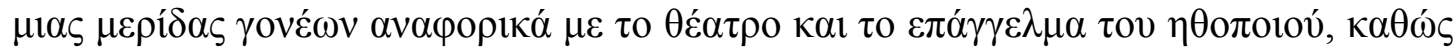

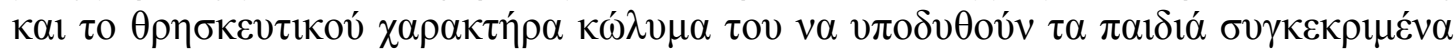

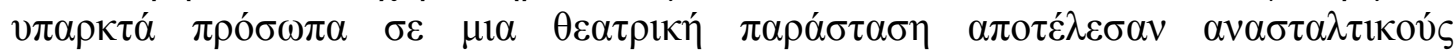

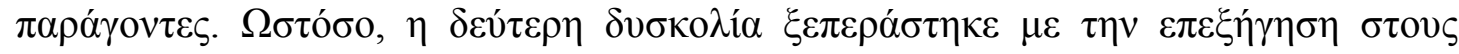

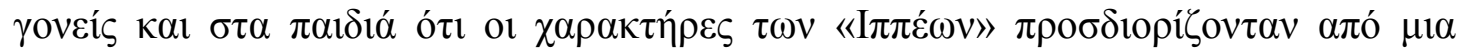

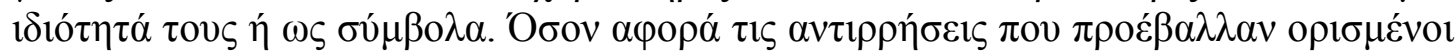

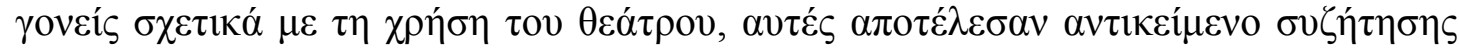

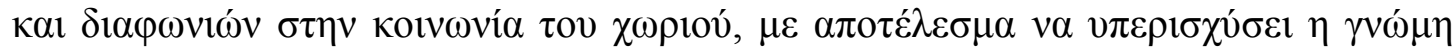

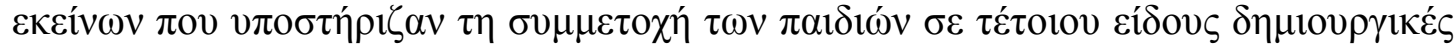
$\delta \rho \alpha \sigma \tau \eta \rho \dot{\tau} \tau \tau \varepsilon \varsigma$.

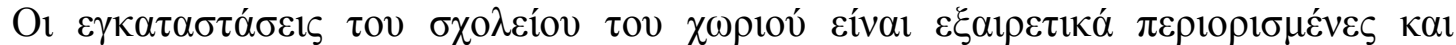

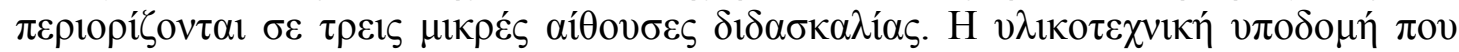

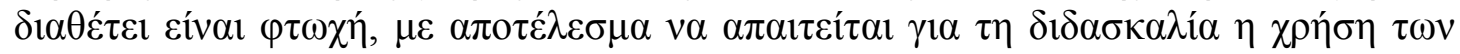

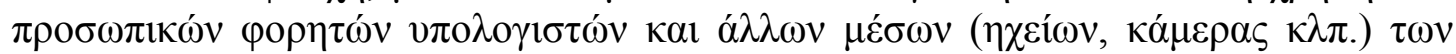

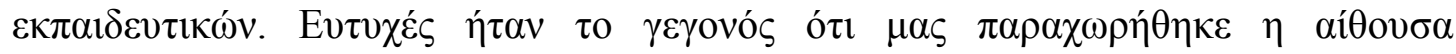

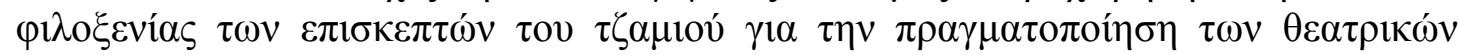

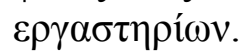

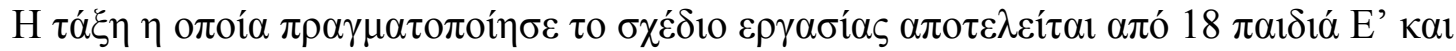

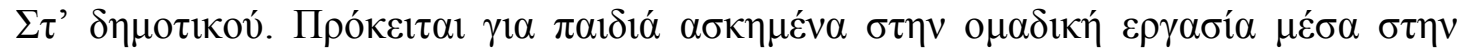

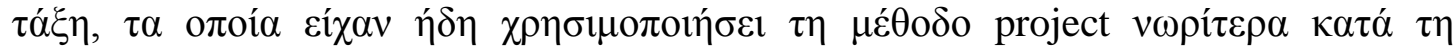

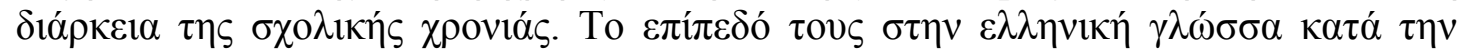

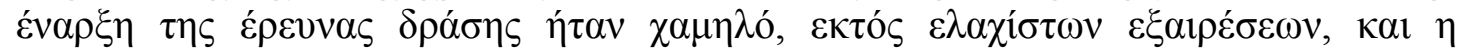

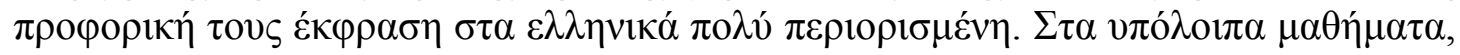

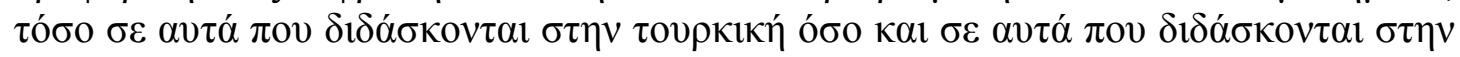

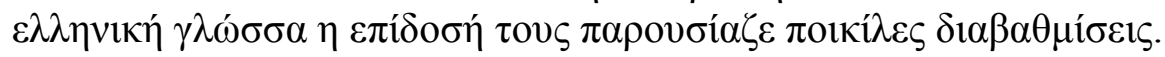

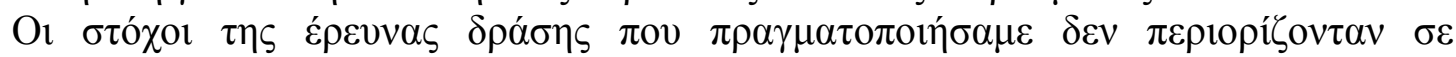

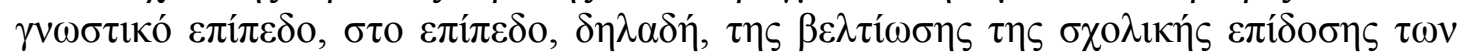

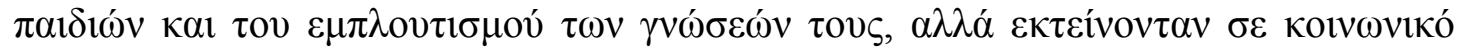

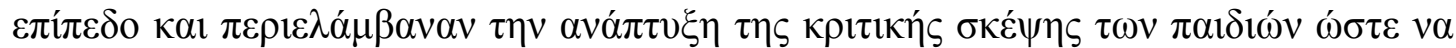

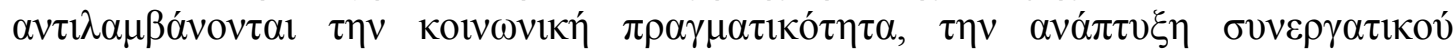

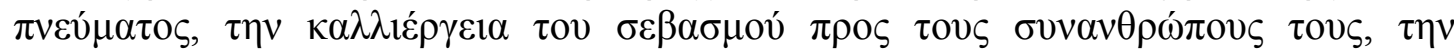




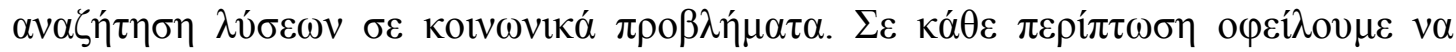

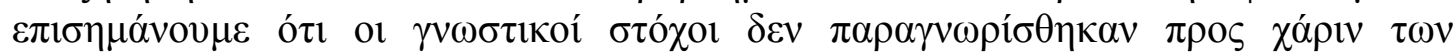

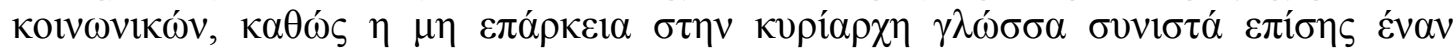

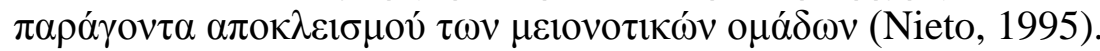

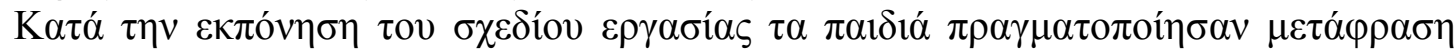

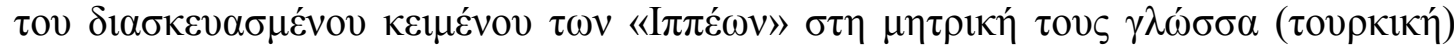

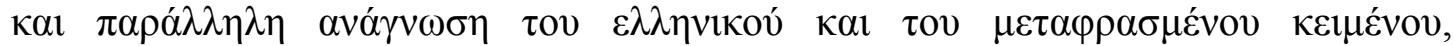

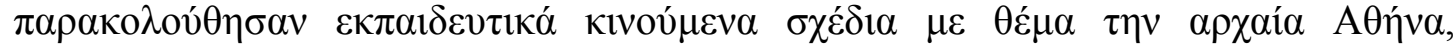

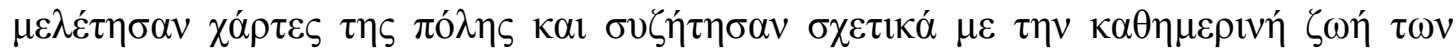

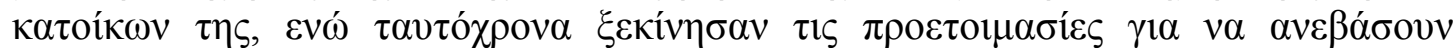

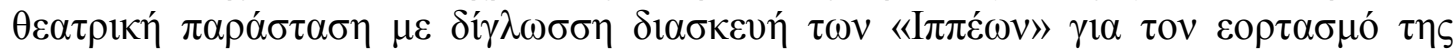

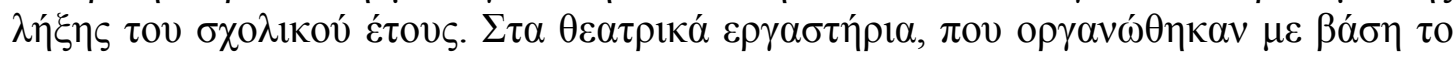

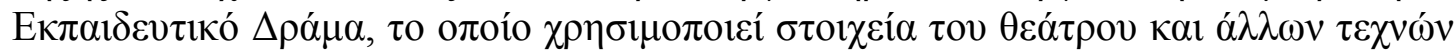

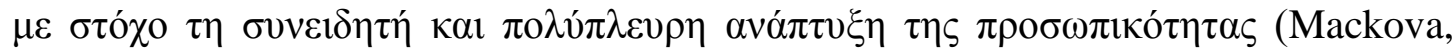

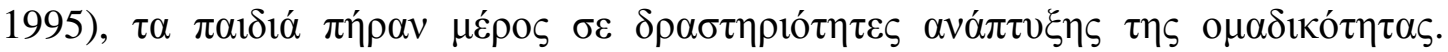

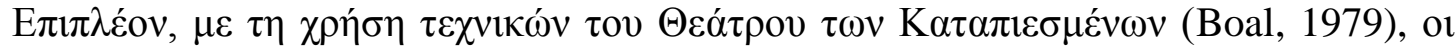

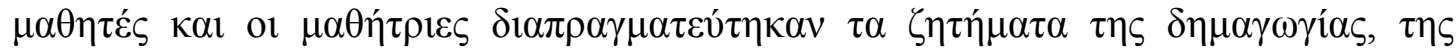

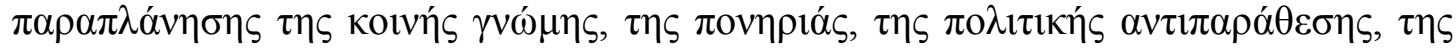

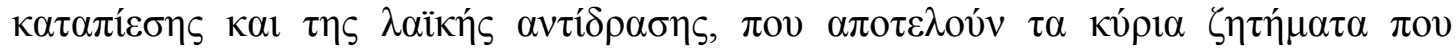

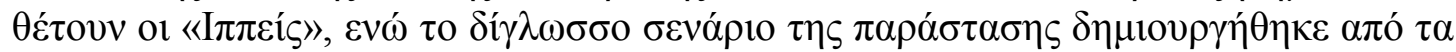

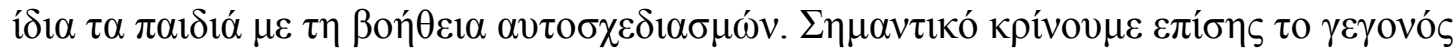

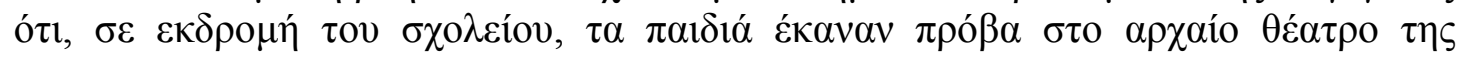

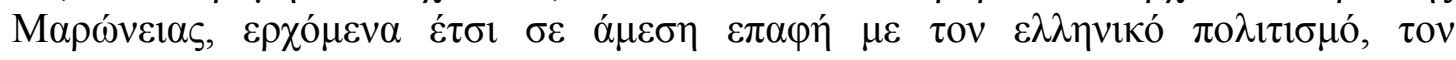

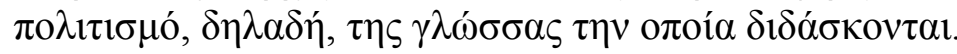

\section{$\Sigma v \mu \pi \varepsilon \rho \alpha ́ \sigma \mu \alpha \tau \alpha$}

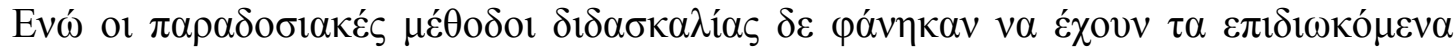

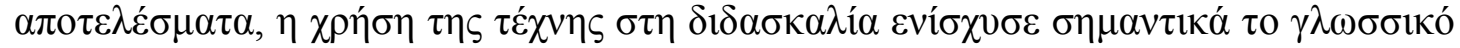

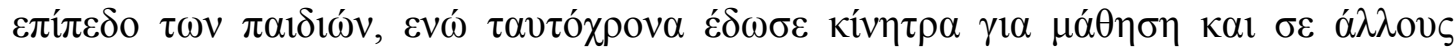

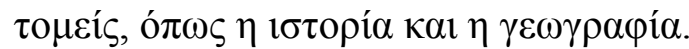

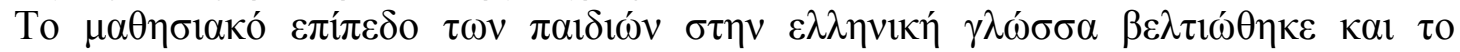

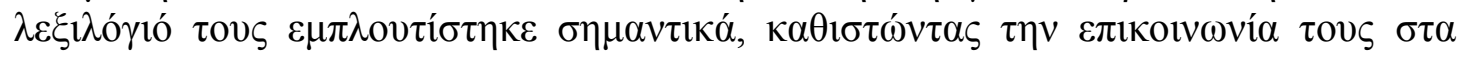

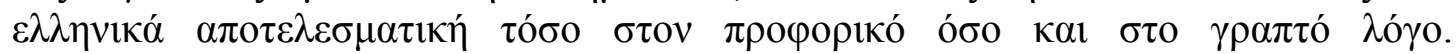

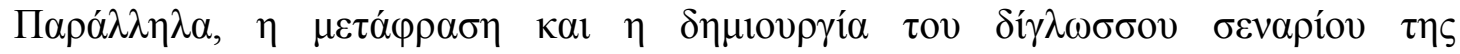

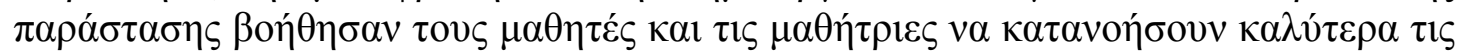

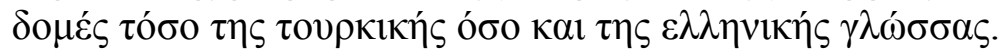

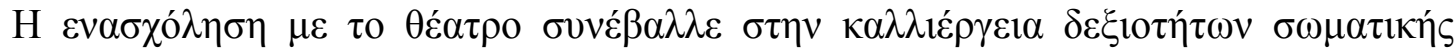

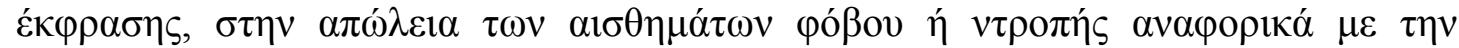

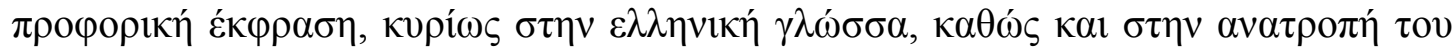

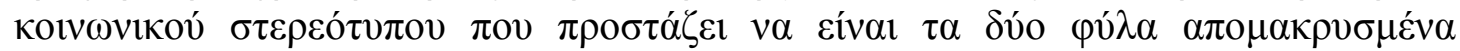

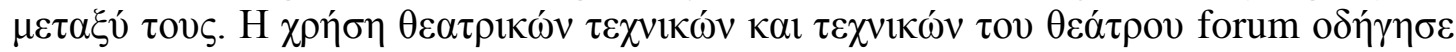

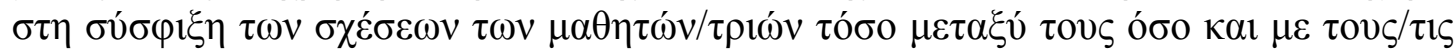

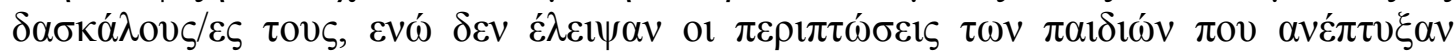

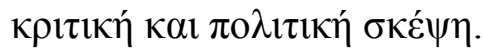

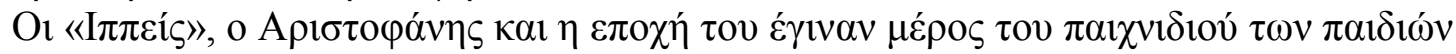

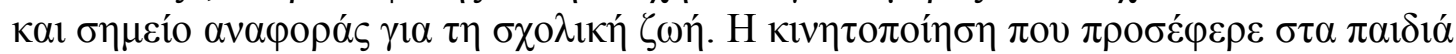

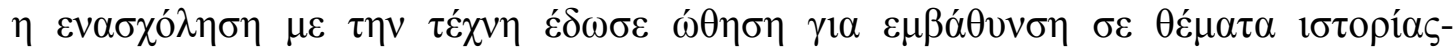

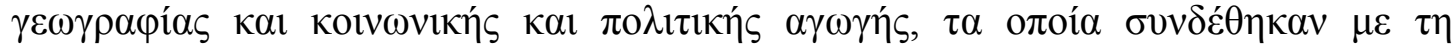

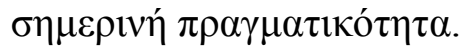




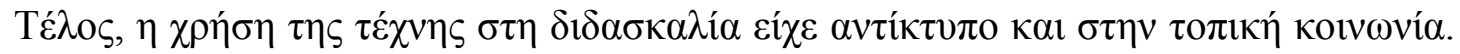

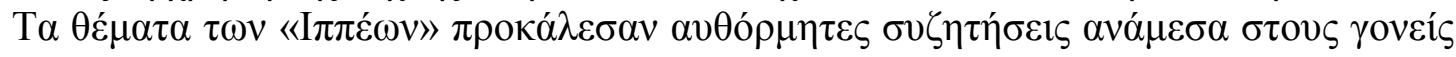

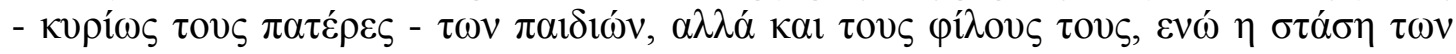

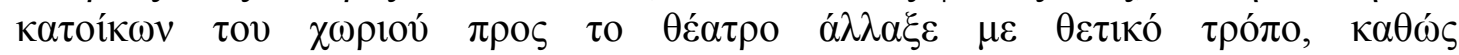

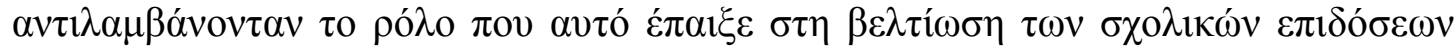
$\tau \omega \nu \pi \alpha 1 \delta i \omega ́ v \tau o v s$.

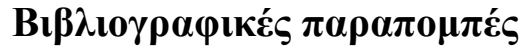

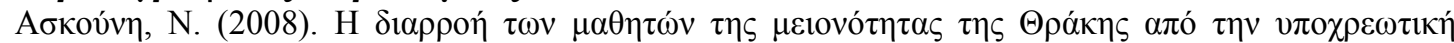

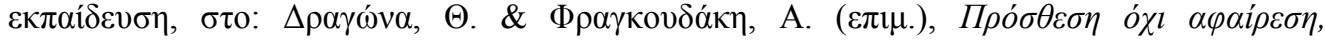

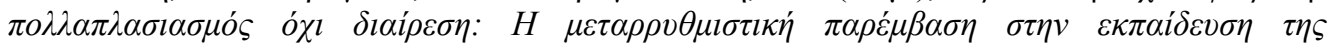

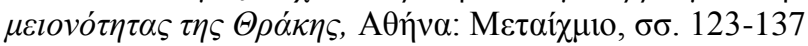

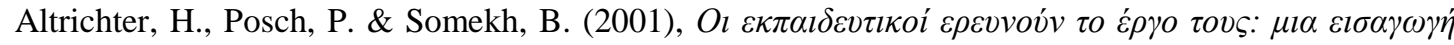

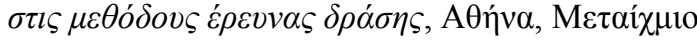

Boal, A. (1979), Theatre of the Oppressed, London, Pluto Press

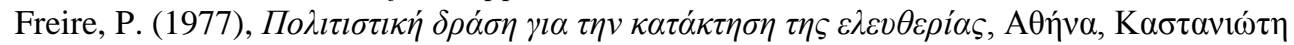

Freire, P. (1996), Pedagogy of the oppressed, London: Penguin Giroux, H.A. (1992), Border crossings: cultural workers and the politics of education, New York, Routledge

Giroux, H.A. (1992), Border crossings: cultural workers and the politics of education, New York, Routledge

Grant, C. (2005), Г

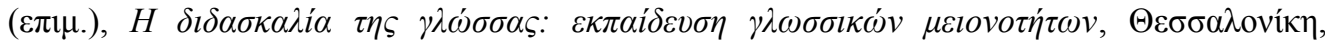

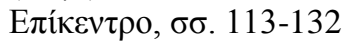

hooks, b. (1994), Teaching to transgress, New York, Routledge

Mackova, S. (1995), Dramaticka Vychova Ve Skole, Brno, Difa JAMU

Nieto, S. (1995), From Brown heroes and holidays to assimilationist agendas: reconsidering the critiques of multicultural education, in: Sleeter, C. \& McLaren, P. (eds.), Multicultural education, critical pedagogy and the politics of difference, New York: State University of New York Press, pp. 191-220

Usher, R. \& Edwards, R. (1994), Postmodernism and education, London, Routledge 